$>$ Les récepteurs activés par les proliférateurs de peroxysomes (PPAR) sont des facteurs de transcription qui appartiennent à la superfamille des récepteurs nucléaires. Trois gènes, répartis sur des chromosomes différents, ont été identifiés: PPAR $\alpha, \operatorname{PPAR} \beta / \delta$ et PPAR $\gamma$. La protéine PPAR $\alpha$ joue un rôle clé dans le contrôle du métabolisme et de l'homéostasie lipidiques. PPAR $\beta / \delta$ est une isoforme ubiquitaire, qui est fortement impliquée dans la physiologie des muscles. Les récepteurs PPAR $\beta / \delta$ et PPAR $\gamma$ sont des facteurs importants pour le développement et le fonctionnement du placenta, et pour l'implantation de l'embryon. PPAR $\gamma$ est un partenaire capital du processus d'adipogenèse. Les PPAR codent aussi des protéines qui interviennent à des degrés divers dans les processus de prolifération, de différenciation et d'apoptose cellulaires. Assez curieusement, le rôle de ces facteurs transcriptionnels dans les processus d'interactions entre cellules et/ ou entre cellules et matrice extracellulaire est rarement souligné, exception faite de leur action anti-inflammatoire. Pourtant, via des mécanismes souvent indirects et incomplètement élucidés, de nouveaux rôles des PPAR se dessinent, qui pourraient, s'ils se précisent, élargir encore la notion de cible thérapeutique associée à ces récepteurs. <

\section{PPAR \\ et interactions \\ des cellules \\ entre elles \\ ou avec la matrice extracellulaire}

Hossam Murad, Cathy Fiatte,

Emilie Brunner, Cécile Huin-Schohn, Hervé Schohn,

Philippe Bécuwe, Lionel Domenjoud, Michel Dauça

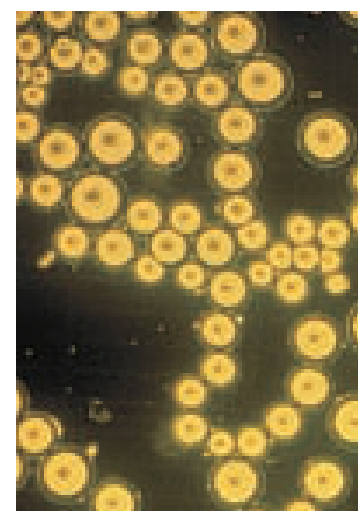

EA3446 «Proliférateurs

de Peroxysomes »,

Faculté des Sciences, BP239,

54506 Vandœuvre-les-Nancy,

France.

Michel.Dauca@

scbiol.uhp-nancy.fr

où se trouve précisément la cadhérine- $\varepsilon$. Cela suggère l'existence d'un lien entre les voies de signalisation de PPAR $\gamma$ et du système cadhérine $\varepsilon / \beta$-caténine. Par ailleurs, il a été récemment montré que l'acide valproïque, un activateur de $\operatorname{PPAR} \beta / \delta$, tératogène in vivo chez I'homme et la souris, est capable de moduler l'expression de la molécule NCAM (neural cell adhesion molecule) [2]. $\operatorname{PPAR} \beta / \delta$ est normalement exprimé dans les cellules F9 de carcinome embryonnaire et son activation par l'acide valproïque induit la différenciation de ces cellules. Sa surexpression dans les cellules $F 9$ traitées par l'acide valproïque induit une forte expression du gène codant NCAM, favorisant ainsi l'adhérence et la différenciation des cellules F9. Réciproquement, la répression de l'activité de $\operatorname{PPAR} \beta / \delta$ inhibe ces effets [2]. En revanche, l'activation de PPAR $\alpha$ ou de PPAR $\gamma$ n'a pas d'effet sur l'expression de NCAM [2]. II est possible que la modulation de l'expression du gène NCAM par PPAR $\beta / \delta$ soit indirecte car aucun élément de réponse ou PPRE (peroxisome proliferator responsive element) aux PPAR n'a été actuellement identifié dans le promoteur du gène NCAM.

\section{Expression de la sémaphorine 6B}

Les PPAR se fixent sur un PPRE situé en amont du gène (HSA)SEMA6B qui code une sémaphorine transmembra- 
naire [3]. Les sémaphorines sont des protéines sécrétées ou transmembranaires qui participent au guidage du cône axonal des neurones, à la régénération axonale, ainsi qu'au développement du tissu nerveux et d'autres tissus [4]. L'expression du gène codant la sémaphorine 6B est réprimée en réponse à l'activation des différents PPAR non seulement dans les cellules T98-G provenant d'un glioblastome, mais aussi dans les cellules MCF-7 qui dérivent d'un adénocarcinome mammaire humain [5]. Ce contrôle négatif de l'expression du gène (HSA)SEMA6B est accentué lorsque les cellules sont traitées à la fois par l'un des agonistes des PPAR et par l'acide 9-cis-rétinoïque qui active RXR, le partenaire des PPAR [5]. Les données concernant NCAM et les sémaphorines suggèrent une implication des PPAR dans la différenciation cellulaire et dans le développement du système nerveux (Figure 1).

\section{Rôle des PPAR dans les processus inflammatoires}

La participation de PPAR $\alpha$ au contrôle des mécanismes anti-inflammatoires, et celle de PPAR $\gamma$ à l'inhibition de la production des cytokines inflammatoires sont désormais bien connues. En revanche, le rôle des PPAR dans les interactions cellulaires associées aux processus inflammatoires mérite une attention particulière. L'infiltration des tissus par les leucocytes est une caractéristique commune à la plupart des maladies inflammatoires chroniques. L'augmentation de l'expression des molécules d'adhérence dans les cellules endothéliales joue un rôle essentiel dans le développement des états inflammatoires en permettant le recrutement des leucocytes, tout particulièrement des lymphocy- tes, et en favorisant leur déplacement, leur adhérence et leur extravasation. L'expression constitutive par les cellules endothéliales humaines d'un PPAR $\gamma$ mutant, actif même en l'absence de ligand, diminue le taux des transcrits codant les protéines VCAM-1 (vascular cell adhesion molecule-1), ICAM-1 (intercellular adhesion molecule-1) et sélectine $\varepsilon$. Elle réprime aussi l'activité des facteurs pro-inflammatoires $N F-\kappa B$ (nuclear factor $-\kappa B$ ) et $A P-1$ (activator protein-1) [6]. L'activation de PPAR $\gamma$ par des ligands naturels ou synthétiques inhibe in vitro l'expression de VCAM-1 à la surface des cellules endothéliales, les empêchant d'interagir avec les monocytes [7, 8]. La pioglitazone, une thiazolidinédione, réduit l'expression des récepteurs CDIlb/CD18 de la membrane plasmique des leucocytes, s'opposant ainsi à leur contact avec les cellules endothéliales [8]. L'induction exercée in vitro par le TNF- $\alpha$ (tumor necrosis factor- $\alpha$ ) sur l'expression de VCAM-1, ICAM-1 (le ligand de CD1lb/CD18), MAdCAM-1 (mucosal adressin cell adhesion molecule-1) et sélectine $\varepsilon$ dans les cellules endothéliales est inhibée par la troglitazone, une thiazolidinédione qui active PPAR $\gamma$ [9]. Le MCC-555, une thiazolidinédione particulière capable d'activer toutes les isoformes de PPAR, diminue fortement la surexpression de VCAM- 1 induite par le TNF $\alpha$ dans les cellules endothéliales car PPAR $\gamma$ activé diminue

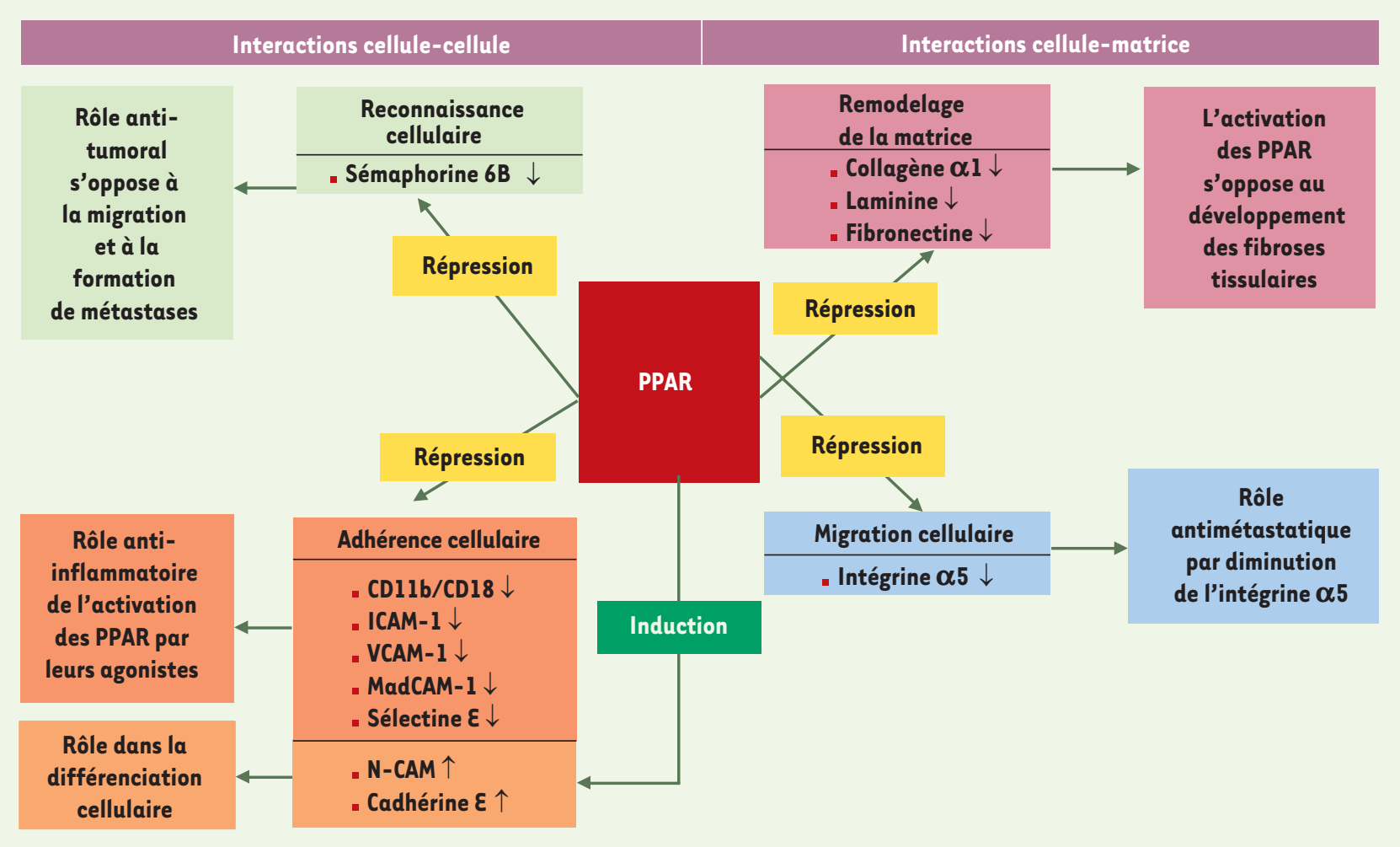

Figure 1. Rôles des PPAR dans les interactions cellule-cellule et cellule-matrice extracellulaire. L'activation des PPAR entraîne soit une augmentation $(\uparrow)$, soit une diminution $(\downarrow)$ de l'expression des molécules. 
la fixation de NF- $\kappa B$ sur le promoteur du gène VCAM-1. Des effets semblables ont été observés avec les ligands de PPAR $\alpha$ et de PPAR $\beta / \delta[7$, 10]. L'activation de PPAR $\gamma$ exerce aussi des effets anti-inflammatoires sur les poumons de rats qui présentent une endotoxémie. Cela se traduit par une diminution de l'expression de ICAM, ce qui réduit l'infiltration des tissus pulmonaires par les neutrophiles [11]. Dans ces situations, l'agoniste de PPAR $\gamma$ empêche le transfert du facteur NF- $\kappa B$ vers le noyau. Le processus inflammatoire qui accompagne les dommages cérébraux chez les rats a également été étudié. L'activation de PPAR $\alpha$ conduit à une baisse substantielle de l'expression de ICAM [12]. D'une façon générale, ces données suggèrent que l'activation des PPAR (tout particulièrement $\operatorname{PPAR} \gamma$ ) peut constituer une approche thérapeutique pour traiter les inflammations. II n'en reste pas moins que ces effets dépendent du type cellulaire, ainsi que de la nature et de la dose de l'agoniste du PPAR. Les PPAR étant impliqués dans des interactions négatives avec NF- $\kappa B$ [13], ils déclenchent une baisse d'expression de plusieurs molécules d'adhérence cellulaire, empêchant ainsi le recrutement et la migration trans-endothéliale des lymphocytes (Figure 1).

\section{PPAR et interactions cellule-matrice extracellulaire}

\section{Expression des protéines matricielles}

Les cellules hépatiques étoilées ( $\mathrm{CH} \varepsilon$ ) synthétisent l'essentiel des composants de la matrice extracellulaire lorsque le foie développe une fibrose. Lors de l'activation des CHE, les activités de leurs métalloprotéinases 2 et 3 augmentent, favorisant le remodelage de la matrice. Les CHE prolifèrent puis surexpriment des protéines matricielles, des inhibiteurs de métalloprotéinases, l'actine- $\alpha$ et les récepteurs des endothélines. Cela conduit à une transformation phénotypique des CHE en myofibroblastes. Durant ces événements, le taux et la capacité de PPAR $\gamma$ à se fixer sur son élément de réponse diminuent fortement, alors que l'inverse se produit pour les facteurs de transcription NF- $\kappa B$ et $A P$ 1. La surexpression ou l'activation de PPAR $\gamma$ dans les CHE entraîne une diminution importante de l'expression de l'actine- $\alpha$ et du collagène $\alpha 1(I)$ et un retour au phénotype classique des CHE [14]. PPAR $\gamma$ réprime la transcription du gène codant le collagène $\alpha 1(\mathrm{I})$ en empêchant la fixation du facteur NF-1 (nuclear factor-1) sur le promoteur de ce gène par compétition avec le co-activateur p300 [15].

L'activation de PPAR $\gamma$ dans des cellules pancréatiques provenant de souris atteintes de pancréatite chronique $[16,17]$, dans des fibroblastes humains pulmonaires [18] et rénaux [19], diminue aussi la synthèse de plusieurs protéines matricielles comme les collagènes ou la laminine (Figure 1). Les ligands de PPARy interviennent également de façon capitale dans l'expression des protéines matricielles lors de I'hypertension artérielle et de l'ischémie du myocarde. Ces situations pathologiques provoquent un remodelage cardiaque qui se traduit par une accumulation surtout de collagène I dans les espaces extracellulaires. La pioglitazone freine la synthèse du collagène I [20].

Par ailleurs, la néphropathie diabétique est caractérisée par une hypertrophie rénale, un épaississement des membranes basales glomérulaires et tubulaires et une accumulation des composants de la matrice extracellulaire sécrétés par les cellules mésangiales. Parmi les protéines matricielles figure la fibronectine. L'expression de cette glycoprotéine est induite par le TGF- $\beta$ (transforming growth factor- $\beta$ ) dans les cellules mésangiales de souris [21] et humaines [22]. Cette induction est inhibée par les ligands de PPAR $\gamma$ qui empêchent la fixation du facteur AP-1 sur l'ADN [21]. Les agonistes de PPAR $\gamma$, qu'ils appartiennent ou non à la famille des thiazolidinédiones, exercent in vitro des effets anti-fibrosants sur les cellules tubulaires rénales HK-2 humaines, exposées à de fortes concentrations de glucose. Ce dernier provoque alors une augmentation de la sécrétion de TGF- $\beta$ ce qui entraîne, par ricochet, une induction de l'expression de la fibronectine. Dans ces cellules rénales exposées à de forts taux de glucose, l'activation de PPAR $\gamma$ diminue la sécrétion de TGF- $\beta$ et réduit fortement l'expression de la fibronectine et du collagène IV. Ces effets résultent de la diminution de l'expression du facteur de transcription AP-1 par PPAR $\gamma$ activé [23].

La capacité d'autres facteurs transcriptionnels comme SPl (pregnancy-specific-beta-1-protein) et CREBP ( $C A M P$ response element binding protein) à se fixer sur le promoteur du gène codant la fibronectine est également diminuée dans les cellules H1838 issues d'un carcinome pulmonaire humain, lorsqu'elles sont traitées par les agonistes de PPAR $\gamma$. Cela entraîne une diminution de l'expression de la fibronectine [24]. Ainsi, dans l'ensemble des pathologies évoquées ci-dessus, l'activation de PPAR $\gamma$ entraîne une diminution de la production de protéines matricielles (Figure 1).

\section{Expression des intégrines}

La fibronectine interagit avec les cellules par l'intermédiaire des intégrines qui sont des glycoprotéines transmembranaires hétérodimériques formées de deux sous-unités, $\alpha$ et $\beta$, associées de façon non covalente. Les intégrines contrôlent les interactions cellules-matrice extracellulaire, l'adhérence et la différenciation des cellules. Ces molécules sont aussi impliquées en pathologie dans le développement de métastases. L'impact de l'activation de PPAR $\gamma$ sur l'expression de l'intégrine $\alpha 5 \beta 1$ a été recherché dans les cellules pulmonaires tumorales humaines car ces cellules expriment fortement cette intégrine lors de la progression tumorale. Les ligands de PPARy déclenchent une diminution de l'expression de la sous-unité $\alpha 5$ de l'intégrine $\alpha 5 \beta 1$ entraînant une diminution de l'adhérence des cellules pulmonaires tumorales à la fibronectine (Figure 1). Parallèlement, l'activation de PPAR $\gamma$ se traduit par une stimulation de la voie de signalisation du facteur ERK (extracellular signal-regulated kinase) et une diminution de la capacité des facteurs transcriptionnels c-jun et Spl à se fixer sur le promoteur du gène $\alpha 5$, ce qui explique la baisse d'expression de ce gène [25]. 


\section{Conclusions}

Ces travaux mettent en lumière le rôle des PPAR dans les interactions cellule-cellule et cellule-matrice extracellulaire. D'une façon générale, l'activation de ces récepteurs nucléaires par des agonistes naturels ou synthétiques a les effets suivants: elle favorise la différenciation cellulaire en augmentant l'expression de molécules d'adhérence comme NCAM et la cadhérine $\varepsilon$; elle joue un rôle antiinflammatoire dans de nombreux tissus en provoquant une baisse d'expression d'autres molécules d'adhérence (ICAM-1, VCAM-1...), ce qui réduit les interactions entre cellules endothéliales et monocytes et l'extravasation de ces derniers; elle s'oppose enfin au développement de fibroses ou à la formation de métastases en freinant les remodelages matriciels et en limitant les capacités de migration des cellules. Ces effets résultent soit d'une action directe des PPAR par fixation sur un PPRE dans la région régulatrice d'un gène cible (c'est le cas pour le gène (HSA)SEMA6B), soit d'une action indirecte en s'opposant notamment à la fixation d'autres facteurs de transcription (NF- $\kappa B, A P-1$, Spl, c-jun, etc.) sur leurs éléments cibles. Si les PPAR sont des acteurs majeurs du métabolisme énergétique cellulaire, leur implication dans les processus d'interactions cellulaires est également importante et représente un domaine de recherche en pleine expansion. $\diamond$

\section{SUMMARY}

PPARs and cell-cell or cell-extracellular matrix interactions

The peroxisome proliferator-activated receptors (PPARs) are transcription factors and belong to the superfamily of nuclear receptors. They are encoded by three genes located on different chromosomes: PPAR $\alpha$, PPAR $\beta / \delta$ and PPAR $\gamma$. PPAR $\alpha$ plays a key role in the control of lipid metabolism and homeostasis. PPAR $\beta / \delta$ is expressed ubiquitously and participates in skeletal muscle physiology. PPAR $\beta / \delta$ and PPAR $\gamma$ are important factors for placental development and function as well as for embryo implantation. In addition, PPAR $\gamma$ is mainly involved in adipogenesis. PPARs also participate more or less to cell proliferation, differentiation and apoptosis. Surprisingly, the involvement of these transcription factors in cell-cell and/or cell-matrix interactions has not yet been reviewed except for their role as therapeutic agents in inflammation. Nevertheless, several pioneer reports have recently provided some new insights in this research field, by suggesting that PPARs are involved, directly or indirectly, in these interactions and that their activation by specific ligands may lead to potential therapeutic approaches. $\diamond$

\section{RéFÉRENCES}

1. Ohta $T$, Elnemr A, Yamamoto $M$, et al. Thiazolidinedione, a peroxisome proliferator-activated receptor-gamma ligand, modulates the $\varepsilon$-cadherin/beta-catenin system in a human pancreatic cancer cell line, BxPC-3. Int J Oncol $2002 ; 21: 37-42$.

2. Lampen A, Grimaldi PA, Nau H. Modulation of peroxisome proliferator-activated receptor delta activity affects neural cell adhesion molecule and polysialyltransferase ST8SialV induction by teratogenic valproic acid analogs in F9 cell differentiation. Mol Pharmacol 2005; $68: 193-203$.

3. Collet $P$, Domenjoud L, Devignes MD, et al. The human semaphorin $6 \mathrm{~B}$ is down regulated by PPARs. Genomics $2004 ; 83: 1141-50$.

4. Bloch-Gallego $\varepsilon$, Sotelo C. Chimio-attraction ou chimio-répulsion axonale : rôle des nétrines et des sémaphorines. Med Sci (Paris) $1998 ; 14: 44-52$.
5. Murad H, Collet P, Huin-Schohn C, et al. Effects of PPAR and RXR ligands in semaphorin 6B gene expression of human MCF-7 breast cancer cells. Int J Oncol $2006 ; 28: 977-84$.

6. Wang N, Verna L, Chen NG, et al. Constitutive activation of peroxisome proliferator-activated receptor- $\gamma$ suppresses pro-inflammatory adhesion molecules in human vascular endothelial cells. J Biol Chem $2002 ; 277: 34176-81$

7. Jackson SM, Parhami F, Xi XP, et al. Peroxisome proliferator-activated receptor activators target human endothelial cells to inhibit leukocyteendothelial cell interaction. Arterioscl Thromb Vasc 1999; 19 : 2094-104.

8. Imamoto $\varepsilon$, Yoshida N, Uchiyama K, et al. Inhibitory effect of pioglitazone on expression of adhesion molecules on neutrophils and endothelial cells. Biofactors $2004 ; 20: 37-47$.

9. Sasaki M, Jordan P, Welbourne T, et al. Troglitazone, a PPAR- $\gamma$ activator prevents endothelial cell adhesion molecule expression and lymphocyte adhesion mediated par TNF- $\alpha$. BMC Physiol $2005 ; 5: 3$.

10. Kurebayashi S, Xu X, Ishii S, et al. A novel thiazolidinedione MCC -555 down-regulates tumor necrosis factor- $\alpha$-induced expression of vascular cell adhesion molecule- 1 in vascular endothelial cells. Atherosclerosis $2005 ; 182: 71-7$

11. Lieu D, Zeng BX, Zhang SH, et al. Rosiglitazone, an agonist of peroxisome proliferator-activated receptor $\gamma$, reduces pulmonary inflammatory response in a rat model of endotoxemia. Inflamm Res $2005 ; 54: 464-70$.

12. Besson VC, Chen XR, Plotkine $M$, et al. Fenofibrate, a peroxisome proliferator-activated receptor $\alpha$ agonist, exerts neuroprotective effects in traumatic brain injury. Neurosci Lett $2005 ; 388$ : 7-12.

13. Vlaeminck-Guillem V, Laudet V, Duterque-Coquillaud M. Negative crosstalk between nuclear receptors and transcription factors: implications in inflammation and oncogenesis. Med Sci (Paris) 2003 ; 19 : 1121-7.

14. Hazra S, Miyahara T, Rippe RA, et al. PPAR gamma and hepatic stellate cells. Comp Hepatol $2004 ; 3$ : 57-9.

15. Yavrom S, Chen L, Xiong S, et al. Peroxisome proliferator-activated receptor $\gamma$ suppresses proximal $\alpha l(I)$ collagen promoter via inhibition of p300-facilitated NF-I binding to DNA in hepatic stellate cells. J Biol Chem $2005 ; 280$ : 40650-9.

16. Masamune A, Kikuta K, Satoh M, et al. Ligands of peroxisome proliferator-activated receptor $\gamma$ block activation of pancreatic stellate cells. J Biol Chem $2002 ; 277$ : 141-7.

17. Van Westerloo DJ, Florquin $S$, de Boer AM, et al. Therapeutic effects of troglitazone in experimental chronic pancreatitis in mice. Am J Pathol $2005 ; 166: 721-8$.

18. Burgess HA, Daugherty $L E$, Thatcher TH, et al. PPAR $\gamma$ agonists inhibit TGF- $\beta$ induced pulmonary myofibroblast differentiation and collagen production : implications for therapy of lung fibrosis. Am J Physiol Lung Cell Mol Physiol 2005 ; 288 : L1146-53.

19. Zafiriou S, Stanners SR, Saad S, et al. Pioglitazone inhibits cell growth and reduces matrix production in human kidney fibroblasts. J Am Soc Nephrol 2005 ; $16: 638-45$.

20. Chen K, Chen J, Li D, et al. Angiotensin II regulation of collagen type I expression in cardiac fibroblasts. Modulation by PPAR- $\gamma$ ligand pioglitazone. Hypertension 2004 ; $44: 655-61$.

21. Guo B, Koya D, Isono M, et al. Peroxisome proliferator-activated receptor- $\gamma$ ligands inhibit TGF- $\beta 1$-induced fibronectin expression in glomerular mesangial cells. Diabetes $2004 ; 53: 200-8$.

22. Maeda A, Horikoshi S, Godha T, et al. Pioglitazone attenuates TGF$\beta 1$-induction of fibronectin synthesis and its splicing variant in human mesangial cells via activation of peroxisome proliferator-activated receptor (PPAR) $\gamma$. Cell Biol Int $2005 ; 29: 422-8$.

23. Panchapakesan U, Sumual C, Pollock CA, et al. PPAR $\gamma$ agonists exert antifibrotic effects in renal tubular cells exposed to high glucose. Am J Physiol Renal Physiol 2005 ; 289 : Fl153-8.

24. Han SW, Ritzenthaler JD, Rivera HN, et al. Peroxisome proliferatoractivated receptor- $\gamma$ ligands suppress fibronectin gene expression in human lung carcinoma cells: involvement of both CRE and Spl. Am J Physiol Lung Cell Mol Physiol 2005 ; 289 : L419-28.

25. Han S, Rivera HN, Roman J. Peroxisome proliferator-activated receptor$\gamma$ ligands inhibit $\alpha 5$ integrin gene transcription in non-small cell lung carcinoma cells. Am J Respir Cell Mol Biol 2005; $32: 350-9$.

\section{TIRÉS À PART}

M. Dauça 